BRAVZULIAN JOURNAL

OF MEDICAL AND BIOLOGICAL RESFARCH

www.bjournal.com.br
ISSN 0100-879X

Volume 43 (5) 381-496 May 2011

BIOMEDICAL SCIENCES

AND

CLINICAL INVESTIGATION

Braz J Med Biol Res, May 2011, Volume 44(5) 477-482

doi: 10.1590/S0100-879X2011007500051

Reduction of the amount of intestinal secretory IgA in fulminant hepatic failure

Dong-yan Liu, Weiguo Jiang and Pei Liu

The Brazilian Journal of Medical and Biological Research is partially financed by

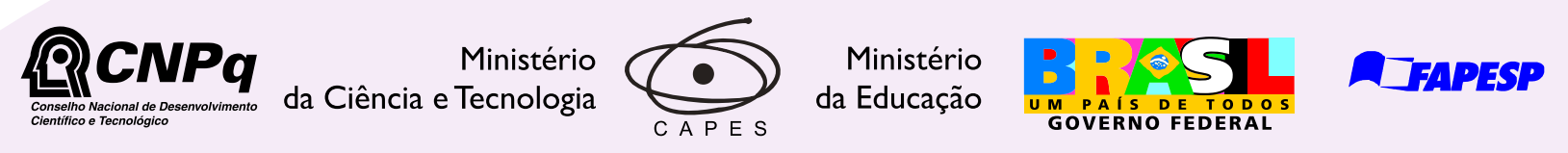

Institutional Sponsors
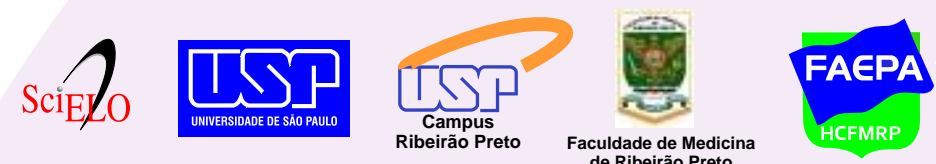

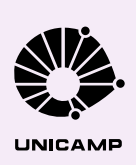

$\oplus$ SHIMADZU

GE Healthcare
Hotsite of proteomics metabolomics developped by:

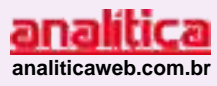




\title{
Reduction of the amount of intestinal secretory $\lg A$ in fulminant hepatic failure
}

\author{
Dong-yan Liu ${ }^{1}$, Weiguo Jiang ${ }^{2}$ and Pei Liu ${ }^{3}$ \\ ${ }^{1}$ Research Center, China Medical University Affiliated Shengjing Hospital and Key Laboratory of \\ Congenital Malformation Research, Ministry of Health, Shenyang, China \\ 2Pathobiology Department, China Medical University Affiliated Shengjing Hospital, Shenyang, China \\ ${ }^{3}$ China Medical University Affiliated Shengjing Hospital, Shenyang, China
}

\begin{abstract}
Intestinal barrier dysfunction plays an important role in spontaneous bacterial peritonitis. In the present study, changes in the intestinal barrier with regard to levels of secretory immunoglobulin A (SIgA) and its components were studied in fulminant hepatic failure (FHF). Immunohistochemistry and double immunofluorescent staining were used to detect intestinal IgA, the secretory component (SC) and SIgA in patients with FHF (20 patients) and in an animal model with FHF (120 mice). Real-time PCR was used to detect intestinal SC mRNA in the animal model with FHF. Intestinal SIgA, IgA, and SC staining in patients with FHF was significantly weaker than in the normal control group (30 patients). Intestinal IgA and SC staining was significantly weaker in the animal model with FHF than in the control groups (normal saline: 30 mice; lipopolysaccharide: 50 mice; D-galactosamine: 50 mice; FHF: 120 mice). SC mRNA of the animal model with FHF at 2, 6 , and $9 \mathrm{~h}$ after injection was $0.4 \pm 0.02,0.3 \pm 0.01$, $0.09 \pm 0.01$, respectively. SC mRNA of the animal model with FHF was significantly decreased compared to the normal saline group $(1.0 \pm 0.02)$ and lipopolysaccharide group $(0.89 \pm 0.01)$. The decrease in intestinal SIgA and SC induced failure of the intestinal immunologic barrier and the attenuation of gut immunity in the presence of FHF.
\end{abstract}

Key words: Fulminant hepatic failure; Secretory IgA; IgA; Secretory component; Intestine

\section{Introduction}

Fulminant hepatic failure (FHF) is a rare but potentially fatal complication of acute hepatitis. Spontaneous bacterial peritonitis (SBP) has been defined as culture positivity with a neutrophilic response $\left(\geq 250\right.$ cells $\left./ \mathrm{mm}^{3}\right)$ in ascitic fluid $(1,2)$. SBP, which occurs in $15-25 \%$ of patients with cirrhosis $(3,4)$, has been reported to occur in FHF $(5-8)$. It has been shown that mortality is 2.5 -fold higher and morbidity 2-fold higher in patients with SBP than in those without SBP (8).

Secretory $\lg \mathrm{A}(\mathrm{S} \lg \mathrm{A})$, which is composed of $\lg \mathrm{A}$, secretory component (SC) and the $\mathrm{J}$ chain, is an important component of the intestinal immunologic barrier. IgA is a predominant Ig in mucosal secretions and serves as the first line of humoral defense on intestinal mucosal surfaces. The majority of these IgA proteins are monomers, while the rest are predominantly polymeric to facilitate efficient transport to the mucosal secretions mediated via the polymeric Ig receptor (PIgR). SC is the extracellular component of the $\mathrm{PIgR}$ responsible for the transcytosis of newly synthesized $\lg A$ (polymeric $\lg A$ ). SIgA and its components, SC and IgA, are important components of the intestinal immunologic barrier. Up to now, investigations of the intestinal barrier in patients with FHF have focused mainly on the mechanical barrier and barrier to the microorganism. In the present study, SIgA, SC and $\lg A$ were measured in patients with $\mathrm{FHF}$ and in an animal model of FHF in order to elucidate the state of the intestinal immunologic barrier.

\section{Material and Methods}

\section{Tissue samples}

Paraffin-embedded sections of intestinal tissue from 20 patients with FHF who had died were obtained from Youan Hospital in Beijing. For controls, paraffin-embedded sections of normal intestine tissue from 30 patients who had died in a traffic accident were obtained from China Medical University, affiliated with Shengjing Hospital. All

Correspondence: Pei Liu, China Medical University Affiliated Shengjing Hospital, Shenyang 110004, China.

E-mail: syliupei2003@yahoo.com.cn

Received August 31, 2010. Accepted March 31, 2011. Available online April 25, 2011. Published May 16, 2011. 
experimental procedures were approved by the Ethics Committee of China Medical University before the beginning of the study.

\section{Animal model of FHF \\ Male BALB/c mice weighing 18-22 g were randomly divided into four groups. group 1 (30 mice): normal saline (NS); group 2 (50 mice): lipopolysaccharide (LPS); group 3 (50 mice): D-galactosamine (D-GalN); group 4 (120 mice): FHF (LPS and GalN; Sigma, USA). Mice were injected intraperitoneally with LPS $(10 \mu \mathrm{g} / \mathrm{kg})$ and/or GalN (800 mg/ $\mathrm{kg}$ ). Mice of group 4 were killed by decapitation 2, 6, 9, 12 , and $24 \mathrm{~h}$ after injection. The NS, LPS and GaIN mice were killed by decapitation $9 \mathrm{~h}$ after the injection of NS, LPS or GalN (9). The study was approved by the animal Ethics Committee of China Medical University.}

\section{Immunohistochemistry of tissue samples}

Intestinal $\lg \mathrm{A}$ and $\mathrm{SC}$ of patients and mice with FHF were determined by immunostaining. Paraffin-embedded intestinal sections of patients and mice with FHF were deparaffinized, rehydrated, and stained with goat anti-human IgA or anti-mouse IgA (Sigma) and mouse anti-human SC (SigA; Sigma) or anti-mouse SC mAb (Bethyl, USA) by sequential incubation. The slides were rinsed three times with 0.02 M PBS, pH 7.4, after each incubation, and sections were counterstained with hematoxylin. The sections from the same patient or the mouse were processed without the primary antibody and then examined by the procedure detailed above as a control for nonspecific binding of the secondary antibody. The absorbance values of IgA and SC were determined with the Image analysis software (China) after scanning.

\section{Double immunofluorescent staining of intestinal SIgA}

Paraffin-embedded intestinal sections of patients with FHF were deparaffinized, rehydrated, and double-stained by immunofluorescence with rhodamine-conjugated goat anti-human IgA (Southern Biotech, USA) and fluoresceinconjugated mouse anti-human SC (SigA; ICN, USA) by sequential incubation. The slides were rinsed three times with 0.02 M PBS, pH 7.4, after each incubation, and sections were counterstained with hematoxylin. Sections from the same patient or the mouse were processed without the primary antibody and then examined using the procedure detailed above as a control for nonspecific binding of the secondary antibody.

\section{Quantitative real-time polymerase chain reaction of intestinal SC mRNA of mice with FHF}

Total RNA was measured as described earlier in Refs. 10,11 . Briefly, total RNA was extracted from mouse gut tissue with FHF using an RNA Mini Kit from Takara (Takara Biotechnology Co., Ltd., China). The quality of extracted
RNA was determined by the number and size of the bands obtained with agarose gel electrophoresis. cDNA was synthesized using 100 ng RNA. The levels of individual RNA transcripts were quantified by the quantitative real-time polymerase chain reaction (PCR). The primers of SC were as follows: PlgR-F: 5'-CAGACATTAGCATGGCAGACTT CAA-3'; PIgR-R: 5'-TGCCGAGTAGGCCATGTCAG-3'; GAPDH-F: 5'-AATGGTGAAGGTCGGTGTG-3'; GAPDH-R: 5'-TGAAGGGGTCGTTGATGG-3'.

The primers and fluorescent probes for SC and standard were purchased from Takara. The PCR conditions were a preliminary cycle at $95^{\circ} \mathrm{C}$ for $10 \mathrm{~s}$, followed by 45 cycles at $95^{\circ} \mathrm{C}$ for $5 \mathrm{~s}$ and at $60^{\circ} \mathrm{C}$ for $20 \mathrm{~s}$, followed by 1 $\min$ at $60^{\circ} \mathrm{C}$ and $5 \mathrm{~s}$ at $95^{\circ} \mathrm{C}$. We also confirmed that the efficiency of amplification for each target gene (GAPDH) was $100 \%$ in the exponential phase of PCR. The levels of SC mRNA and GAPDH mRNA were determined according to the standard (Takara). The mRNA levels were normalized to GAPDH mRNA by dividing SC gene copies of the samples by the SC gene copies of GAPDH. The intestinal RNA level of the NS group was assumed to be 1 , with which the intestinal RNA levels of other groups were compared.

\section{Statistical analysis}

The statistical differences between treatment groups were determined by the $t$-test using the SPSS 10.0 software.

\section{Results}

\section{Intestinal IgA and SC staining in patients}

In the normal control group, SC staining of the cytoplasm and membranes of intestinal epithelial cells was brown (Figure 1A). SC staining of the cytoplasm and of the membranes of intestinal epithelial cells in patients with FHF was pale and considerably weaker than in the normal control group (Figure 1B). IgA staining of the cytoplasm of plasma cells within the lamina propria and of intestinal epithelial cells was brown in the normal control group (Figure 1C). However, in patients with FHF, IgAstaining of the cytoplasm of plasma cells within the lamina propria was pale brown and $\lg A$ staining of intestinal epithelial cells was pale. $\lg A$ staining in patient samples was notably weaker than that of the normal control group (Figure 1D). The mean absorbance values of SC and IgA in patients with FHF were significantly lower than those of the normal control group $(P<0.01$; Figure 1E).

\section{Double immunofluorescence staining}

The results of intestinal double immunofluorescence showed that the cytoplasm of normal intestinal epithelial cells stained orange (SC, SIgA) and the membranes stained yellow (SC, IgA, SIgA), and that the plasma cells within the lamina propria stained orange (SlgA) and yellow (IgA, SlgA; Figure 2A). However, in patients with FHF, the staining of 
the intestinal epithelial cell cytoplasm and membranes as well as plasma cells within the lamina propria was green (Figure 2B). The staining results proved that intestinal SC, $\operatorname{IgA}$ and SIgA were decreased compared to the normal control group.

\section{Intestinal IgA staining in the mouse model of FHF}

$\lg A$ staining of the cytoplasm and/or membranes of intestinal epithelial cells, as well as of plasma cells within the lamina propria, was weaker in the mouse FHF group (Figure 3D) compared to the NS (Figure 3A), LPS (Figure
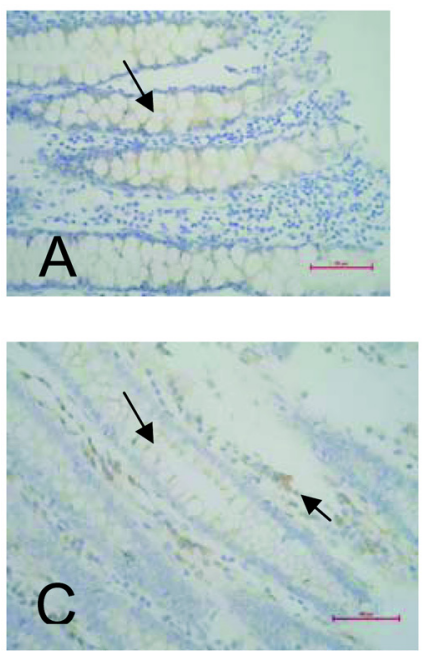
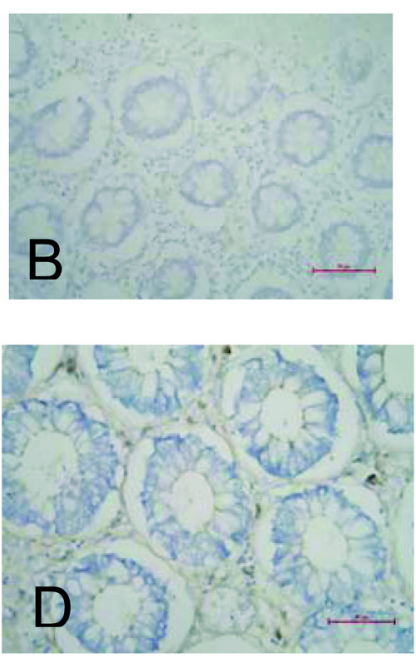

$\mathbf{E}$

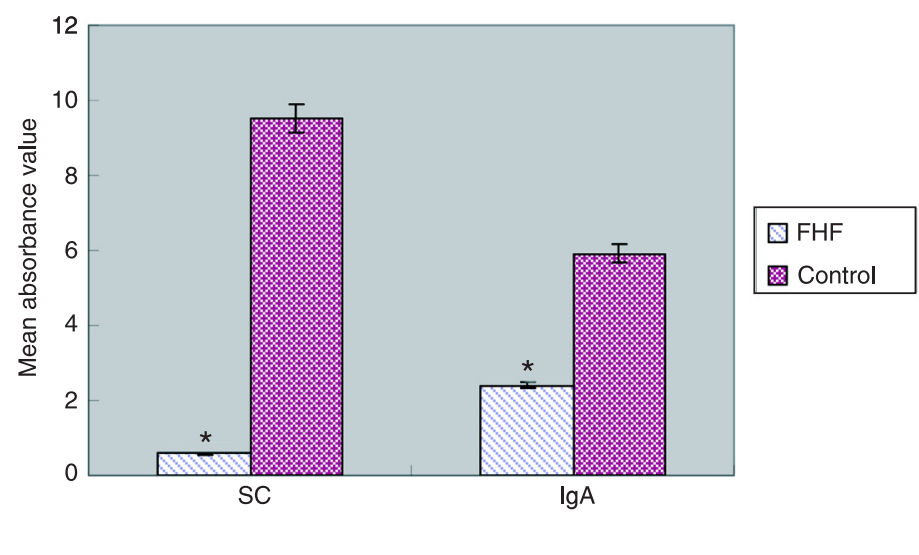

Figure 1. Secretory component (SC) and IgA staining of intestinal epithelial cells in patients with fulminant hepatic failure (FHF). $A, \mathrm{SC}$ staining of intestinal epithelial cells in the normal control group (30 patients) was brown (see arrow). $B, \mathrm{SC}$ staining of intestinal epithelial cells in patients with FHF (20 patients) was pale and remarkably weaker than in the normal control group. The staining results indicated that intestinal SC was significantly decreased in patients with FHF. C, IgA staining of plasma cells within the lamina propria and of intestinal epithelial cells in the normal control group was brown (see arrows). $D$, IgA staining of the cytoplasm of plasma cells within the lamina propria was pale brown and IgA staining of intestinal epithelial cells in patients with FHF was pale and remarkably weaker than in the normal control group. The staining results indicated that intestinal IgA in patients with $\mathrm{FHF}$ was significantly decreased. Magnification bars $=50 \mu \mathrm{m}$. E, The absorbance values of SC and IgA were notably less in patients with FHF compared to the normal control group. Data are reported as means $\pm S D$. ${ }^{*} P<0.01$ compared to the normal control group ( $t$-test). 
3B) and GalN groups (Figure 3C). The mean absorbance values of $\lg A$ in the mouse FHF group were significantly decreased from 2 to $12 \mathrm{~h}$ compared to the NS control group $(P<0.01$; Figure 3E).

\section{Intestinal SC staining in mice with FHF}

SC staining of the cytoplasm and/or the membranes of intestinal epithelial cells was significantly weaker in the mouse FHF group (Figure 4D) compared to the NS group (Figure 4A), LPS group (Figure $4 B$ ), and GalN group (Figure 4C). The mean absorbance values of $\mathrm{SC}$ in the mouse FHF group were significantly decreased from 2 to $12 \mathrm{~h}$ compared to the NS group $(\mathrm{P}<0.01$; Figure 4E).

\section{SC mRNA of intestinal tissues} in the animal model of FHF

The relative content of intestinal SC mRNA in mice with FHF at 2, 6, and $9 \mathrm{~h}$ after injection was $0.4 \pm 0.02,0.3 \pm 0.01,0.09$ \pm 0.01 , respectively. In the mice with FHF, intestinal SC mRNA was significantly lower at 2 to 9 h compared to the NS group (1.0 $\pm 0.02)$ and LPS group (0.89 \pm 0.01 ; $P<0.01)$. In the GalN group, intestinal SC mRNA $(0.2 \pm 0.00)$ was also lower compared to the NS group and LPS group ( $P$ < 0.01; Figure 5).

\section{Discussion}

SBP is a common illness in patients with cirrhosis and ascites, which occurs without any apparent focus of infection. Bacterial translocation, gut motility alterations, and intestinal barrier dysfunction, along with bacterial overgrowth and changes in intestinal permeability play important roles in SBP, and it is evident from a number of studies that the gut is a major source of these bacteria (12). Intestinal SIgA levels are inversely correlated with bacterial overgrowth, bacterial translocation and changes in intestinal permeability (13). SIgA may weaken bacterial translation and restrict bacteria through intestinal
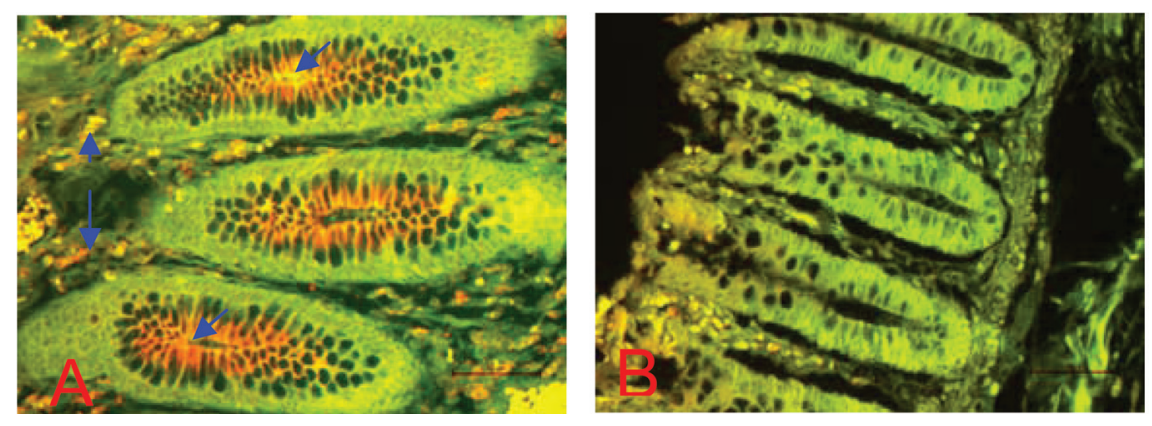

Figure 2. $\lg A$ and secretory $\lg A(S \lg A)$ staining of the intestinal epithelium by double immunofluorescence. $A$, The secretory component (SC) and SIgA stained orange in the cytoplasm and $\mathrm{SC}, \operatorname{IgA}$ and $\mathrm{SIgA}$ on the membranes stained yellow in the normal intestinal epithelial cells. SIgA of the plasma cells within the lamina propria stained orange. IgA and SIgA of the plasma cells within the lamina propria stained yellow (see blue arrows). $B$, In patients with fulminant hepatic failure (FHF), the color of the intestinal epithelial cell cytoplasm, membranes and plasma cells within the lamina propria was green. The staining results indicated that intestinal SC, IgA and SIgA were notably decreased compared to the normal control group. Magnification bars $=50 \mu \mathrm{m}$.

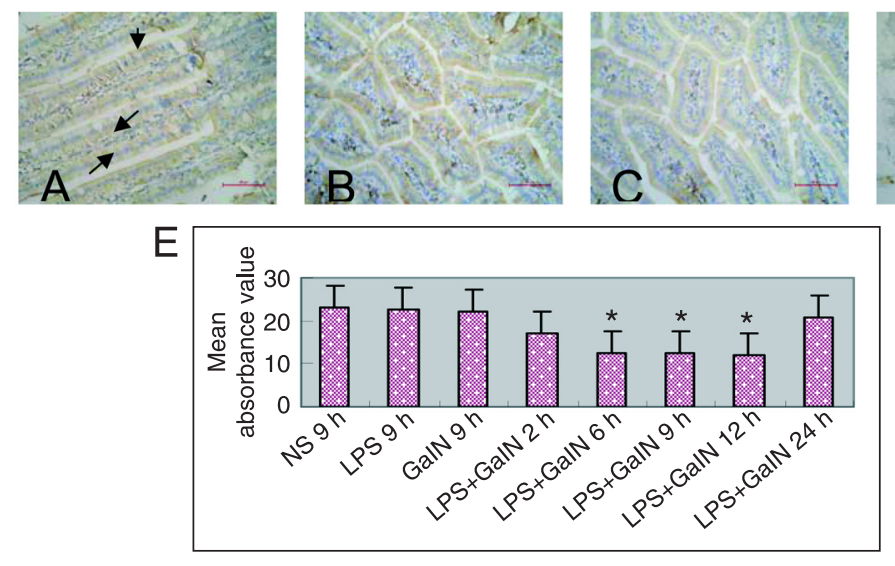

Figure 3. IgA staining of the intestinal epithelium in the animal model with fulminant hepatic failure (FHF). A, In the normal saline (NS) group (30 mice), IgA staining of intestinal epithelial cell cytoplasm, membranes and plasma cells within the lamina propria was deep brown (arrows). $B$, In the lipopolysaccharide (LPS) group (50 mice), IgA staining of intestinal epithelial cell cytoplasm, membranes and plasma cells within the lamina propria was brown. $C$, In the galactosamine (GalN) group (50 mice), IgA staining of the intestinal epithelial cell cytoplasm, membranes and plasma cells within the lamina propria was pale brown. $D$, In the FHF group (120 mice), IgA staining of some intestinal epithelial cell cytoplasm and membranes was pale. Magnification bars $=50 \mu \mathrm{m}$. $E$, The absorbance values of IgA in the animal model with FHF (2, 6,9 , and $12 \mathrm{~h}$ ) were significantly decreased compared to the normal saline (NS), lipopolysaccharide (LPS) and galactosamine (GaIN) groups. Data are reported as means $\pm S D$. ${ }^{*} P<0.01$ compared to NS $9 \mathrm{~h}$, LPS $9 \mathrm{~h}$, and GalN $9 \mathrm{~h}$ (t-test). 
parasites (22) as well as playing a critical role in the immunologically mediated neutralization of cholera toxin (23). In vitro, free SC has been shown to fix the complement component $\mathrm{C} 3 \mathrm{~b}$, suggesting a role for SC in enhancing local immune responses (24).

In liver diseases, notably alcoholic cirrhosis of the liver, intestinal SIgAis decreased (25). In the present study, intestinal $\lg A, S C$ and SIgA were markedly decreased in patients with FHF, this possibly being the reason why gut immunity is weakened in patients with FHF. Changes in intestinal SIgA, IgAand $\mathrm{SC}$ in the animal model of FHF were consistent with those of the patients with FHF. SC mRNAwas simultaneously detected by real-time PCR. A decrease in intestinal SC mRNA was observed in the animal model with FHF. These results confirm that at the mRNAand protein levels, SC is decreased in the animal model of FHF. It has been reported that in
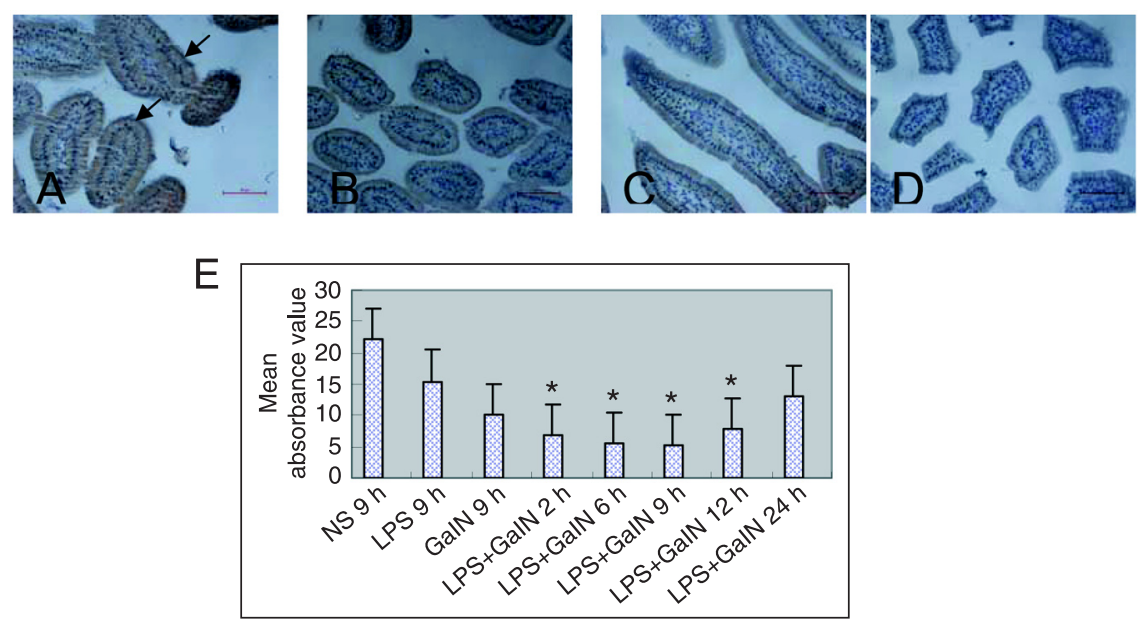

Figure 4. Staining of the secretory component (SC) of the intestinal epithelium in the mouse model of fulminant hepatic failure (FHF). A, In the normal saline (NS) group (30 mice), the SC staining of the intestinal epithelial cell cytoplasm and membranes was deep brown (see arrows). $B$, In the lipopolysaccharide (LPS) group (50 mice), SC staining of the intestinal epithelial cell cytoplasm and membranes was brown. $C$, In the galactosamine (GalN) group (50 mice), SC staining of the intestinal epithelial cell cytoplasm and membranes was pale brown. $D$, In the FHF group (120 mice), SC staining of intestinal epithelial cells was pale. $E$, The absorbance values of SC in the animal model with $\operatorname{FHF}(2,6,9$, and $12 \mathrm{~h})$ were significantly decreased compared to the normal saline (NS), lipopolysaccharide (LPS) and galactosamine (GalN) groups. Data are reported as means \pm SD. ${ }^{*} \mathrm{P}<0.01$ compared to NS $9 \mathrm{~h}$, LPS $9 \mathrm{~h}$, and GalN 9 h ( $t$-test).

mildly inflamed ulcerative colitis le-

sions, SC levels showed no change but tended to decrease with increasing degree of inflammation. Decreased SC expression thus seemed to be related to intensified inflammatory activity (26). Therefore, we think that in patients with FHF, damaged intestinal mucus results in lower levels of intestinal SC and SIgA. Malnourished animals exhibit low levels of hepatic and intestinal plgR. The concentration of biliary SC was also low in malnourished animals (27). In patients with FHF, the disease is usually complicated by malnutrition. Therefore, malnutrition may cause low concentrations of intestinal SC in FHF.

In conclusion, intestinal $\lg \mathrm{A}$ and $\mathrm{SC}(\mathrm{S} \lg \mathrm{A})$ were lower in patients with $\mathrm{FHF}$, with consequent failure of the intestinal immunologic barrier and attenuation of gut immunity.

\section{Acknowledgments}

Research supported by the National Natural Science Foundation of China (\#30670947, \#30871158) and the Education Department Foundation of Liaoning Province (\#05L466, \#2008783).

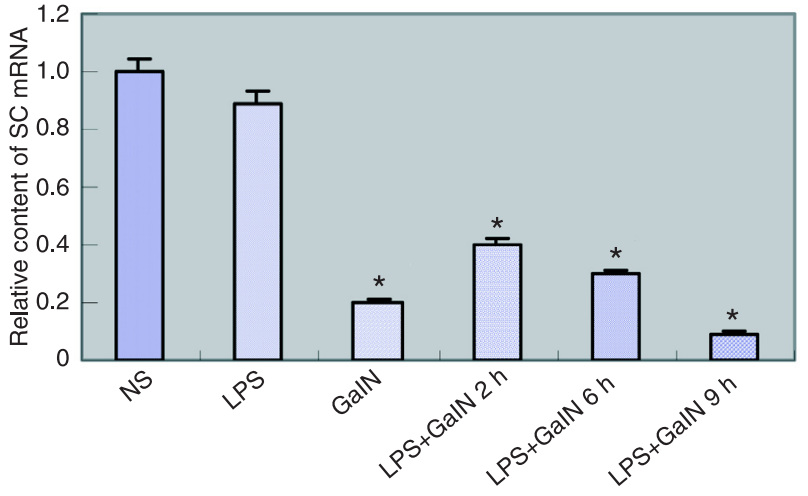

Figure 5. Relative content of the intestinal secretory component (SC) mRNA. Relative content of intestinal SC mRNA in the animal model with fulminant hepatic failure (FHF) was significantly lower compared to the normal saline (NS) and lipopolysaccharide (LPS) groups. The relative content of intestinal SC mRNA in the galactosamine (GalN) group was also lower compared to the NS and LPS groups. Data are reported as means $\pm \mathrm{SD}$. ${ }^{*} \mathrm{P}<0.01$ compared to NS and LPS ( $t$-test). 


\section{References}

1. Chu CM, Chiu KW, Liaw YF. The prevalence and prognostic significance of spontaneous bacterial peritonitis in severe acute hepatitis with ascites. Hepatology 1992; 15: 799803.

2. Valla D, Flejou JF, Lebrec D, Bernuau J, Rueff B, Salzmann $\mathrm{JL}$, et al. Portal hypertension and ascites in acute hepatitis: clinical, hemodynamic and histological correlations. Hepatology 1989; 10: 482-487.

3. Pinzello G, Simonetti RG, Craxi A, Di Piazza S, Spano C, Pagliaro L. Spontaneous bacterial peritonitis: a prospective investigation in predominantly nonalcoholic cirrhotic patients. Hepatology 1983; 3: 545-549.

4. Runyon BA. Spontaneous bacterial peritonitis: an explosion of information. Hepatology 1988; 8: 171-175.

5. Thomas FB, Fromkes JJ. Spontaneous bacterial peritonitis associated with acute viral hepatitis. J Clin Gastroenterol 1982; 4: 259-262.

6. Poddar U, Chawla Y, Dhiman RK, Vaiphei K, Vasishta RK, Dilawari JB. Spontaneous bacterial peritonitis in fulminant hepatic failure. J Gastroenterol Hepatol 1998; 13: 109-111.

7. Dhiman RK, Makharia GK, Jain S, Chawla Y. Ascites and spontaneous bacterial peritonitis in fulminant hepatic failure. Am J Gastroenterol 2000; 95: 233-238.

8. Poddar U, Thapa BR, Prasad A, Sharma AK, Singh K. Natural history and risk factors in fulminant hepatic failure. Arch Dis Child 2002; 87: 54-56.

9. Dongyan Liu, Peng Ding, Pei Liu. The change of secretory IgA of gut induced by TNF- $\alpha$ in liver necrosis mice. Zhong hua Gan zang Bing Za Zhi 2010; 18: 306-308.

10. Schneeman TA, Bruno ME, Schjerven $H$, Johansen FE, Chady L, Kaetzel CS. Regulation of the polymeric Ig receptor by signaling through TLRs 3 and 4: linking innate and adaptive immune responses. J Immunol 2005; 175: 376384.

11. Bruno ME, Kaetzel CS. Long-term exposure of the HT-29 human intestinal epithelial cell line to TNF causes sustained up-regulation of the polymeric Ig receptor and proinflammatory genes through transcriptional and posttranscriptional mechanisms. J Immunol 2005; 174: 7278-7284.

12. Ramachandran A, Balasubramanian KA. Intestinal dysfunction in liver cirrhosis: Its role in spontaneous bacterial peritonitis. J Gastroenterol Hepatol 2001; 16: 607-612.

13. Deitch EA, Xu D, Qi L, Berg R. Elemental diet-induced immune suppression is caused by both bacterial and dietary factors. J Parenter Enteral Nutr 1993; 17: 332-336.

14. Macpherson AJ, Slack E. The functional interactions of commensal bacteria with intestinal secretory IgA. Curr Opin Gastroenterol 2007; 23: 673-678.

15. Corthësy B. Secretory immunoglobulin A: well beyond im- mune exclusion at mucosal surfaces. Immunopharmacol Immunotoxicol 2009; 31: 174-179.

16. Nicholas JM, Stephen JF. Secretory IgA: Arresting microbial pathogens at epithelial borders. Immunol Invest 2010; 39: 383-406.

17. Boullier S, Tanguy M, Kadaoui KA, Caubet C, Sansonetti $P$, Corthésy $B$, et al. Secretory IgA-mediated neutralization of Shigella flexneri prevents intestinal tissue destruction by down-regulating inflammatory circuits. J Immunol 2009; 183: 5879-5885

18. Dallas SD, Rolfe RD. Binding of Clostridium difficile toxin A to human milk secretory component. J Med Microbiol 1998; 47: 879-888.

19. de Araujo AN, Giugliano LG. Lactoferrin and free secretory component of human milk inhibit the adhesion of enteropathogenic Escherichia coli to HeLa cells. BMC Microbiol 2001; 1: 25.

20. Giugliano LG, Ribeiro ST, Vainstein MH, Ulhoa CJ. Free secretory component and lactoferrin of human milk inhibit the adhesion of enterotoxigenic Escherichia coli. J Med Microbiol 1995; 42: 3-9.

21. Hammerschmidt S, Talay SR, Brandtzaeg P, Chhatwal GS. SpsA, a novel pneumococcal surface protein with specific binding to secretory immunoglobulin A and secretory component. Mol Microbiol 1997; 25: 1113-1124.

22. Davids BJ, Palm JE, Housley MP, Smith JR, Andersen YS, Martin MG, et al. Polymeric immunoglobulin receptor in intestinal immune defense against the lumen-dwelling protozoan parasite Giardia. J Immunol 2006; 177: 6281-6290.

23. Uren TK, Wijburg OL, Simmons C, Johansen FE, Brandzaeg $\mathrm{P}$, Strugnell RA. Vaccine-induced protection against gastrointestinal bacterial infections in the absence of secretory antibodies. Eur J Immunol 2005; 35: 180-188.

24. Marshall LJ, Perks B, Ferkol T, Shute JK. IL-8 released constitutively by primary bronchial epithelial cells in culture forms an inactive complex with secretory component. J Immunol 2001; 167: 2816-2823.

25. Pelletier G, Briantais MJ, Buffet C, Pillot J, Etienne JP. Serum and intestinal secretory $\operatorname{lgA}$ in alcoholic cirrhosis of the liver. Gut 1982; 23: 475-480.

26. Rognum TO, Brandtzaeg P, Elgjo K, Fausa O. Heterogeneous epithelial expression of class II (HLA-DR) determinants and secretory component related to dysplasia in ulcerative colitis. Br J Cancer 1987; 56: 419-424.

27. Ha CL, Woodward B. Depression in the quantity of intestinal secretory $\lg A$ and in the expression of the polymeric immunoglobulin receptor in caloric deficiency of the weanling mouse. Lab Invest 1998; 78: 1255-1266. 